\title{
Ornithine Lipid Activates Both TLR4 and the non-canonical NLRP3 Inflammasome
}

\author{
Malvina Pizzuto1,2,@, Laura Hurtado-Navarro ${ }^{1, \#, ~ C r i s t i n a ~ M o l i n a-L o p e z ~}{ }^{1, \#,}$ \\ Jalal Soubhye ${ }^{3}$, Michel Gelbcke ${ }^{3}$, Silvia Rodriguez-Lopez ${ }^{1}$, Jean-Marie \\ Ruysschaert ${ }^{2}$, Pablo Pelegrin ${ }^{1,4}$
}

(1) Molecular Inflammation Group, Biomedical Research Institute of Murcia (IMIB-Arrixaca), Murcia, Spain; (2) Laboratoire de Structure et Fonction des Membranes Biologiques, Université Libre de Bruxelles, Brussels, Belgium; (3) Department of Pharmacognosy, Bioanalysis and Drug Discovery, Faculty of Pharmacy, Université Libre De Bruxelles (ULB), Brussels, Belgium; (4) Department of Biochemistry and Molecular Biology B and Immunology, Faculty of Medicine, University of Murcia, 30120 Murcia, Spain

$\left({ }^{\#}\right)$ These authors contributed equally to this work

(@) corresponding author malvina.pizzuto@gmail.com

Running title: Ornithine lipid activates TLR4 and NLRP3

Key words: Ornithine lipid; TLR4; NLRP3; non-canonical inflammasome; caspase-11; nanoparticles; gram-negative bacteria; lipopolysaccharide; inflammation; interleukin-1; ionizable cationic lipid 


\section{Abstract}

Toll-like receptors (TLRs) are proteins that act as the sentinels of mammalian cells by detecting bacteria and viruses motifs such as the phospholipid lipopolysaccharides (LPS) from Gram-negative bacterial membrane, among others. Bacteria like Vibrio cholerae, when grow in phosphate-depleted medium are unable to produce LPS and other phospholipids and therefore increase the synthesis of ornithine lipids (OLs) to keep membrane integrity and survival. We found that, although the huge structural differences between OL and LPS, our immune system is still able to detect OL and trigger immune response through TLR4 and the non-canonical Nucleotide-binding domain and leucine-rich repeatcontaining pyrin protein 3 (NLRP3) inflammasome. Similar to LPS, OL induced TLR4-dependent tumor necrosis factor (TNF)- $\alpha$ secretion and Nuclear Factor (NF)-KB activation and therefore also elicited the priming of the NLRP3 inflammasome. Moreover, incubation of macrophages with OL causes caspasedependent cleavage of gasdermin D (GSDMD) and consequent $\mathrm{K}^{+}$effluxdependent NLRP3 activation and interleukin (IL)-1 $\beta$ secretion.

Results abstract (if needed): In bone-marrow derived murine macrophages (BMDM) from wild type mice, OLs induced hallmarks of NF-KB activation such as TNF- $\alpha$ secretion and the expression of pro-IL-1 $\beta$ and NLRP3. OL induced NF-KB activation dependent on TLR4 as demonstrated by the NF-kB activation measured in HEK293 reporting cells expressing uniquely TLR4 and by the decrease of TNF- $\alpha$ secretion in macrophages caused by antibodies blocking TLR4. OLs also induced IL-1 $\beta$ secretion dependent on cellular $\mathrm{K}^{+}$efflux and NLRP3/caspase-1 inflammasome, as it was blocked by specific inhibitors and absent on NIrp3 $3^{-/-}$, Pycard ${ }^{-/-}$or Casp1/11 $1^{-/-}$macrophages. 


\section{Introduction:}

Toll-like receptors (TLRs) are transmembrane proteins expressed in macrophages, glial and endothelial cells (Barton and Kagan, 2009). In mammals, there are 13 TLRs, each one consecrated to the detection of a family of bacteria or viruses motifs (Gay et al., 2014). The degree of promiscuity of the TLRs is partially limited to the remedying to pathogen heterogeneity or mutations, being TLR able to recognize molecules with different structures but from the same family, for instance dsRNA, CpG motives in DNA, poly-acylated lipopolysaccharides, proteins, etc. The components of the same family share a molecular pattern mostly absent or non-exposed in mammalian organisms and highly present and exposed in pathogens. These components are called pathogen-associated molecular patterns (PAMPs) (Barton and Kagan, 2009; Gay et al., 2014). In the last years, it has emerged that the interaction made by the pattern can be mimicked by synthetic molecules with apparently different structure (Pizzuto et al., 2016, 2019), and also that molecules lacking a clearly defined pathogen pattern can be recognized by TLR through different binding sites. This is the case for Nickel, Cobalt and cationic ionizable dialkylated lipids that bind at the dimeric interface of TLRs causing TLR4 dimerization and activation (Schmidt et al., 2010; Raghavan et al., 2012; Lonez et al., 2015; Pizzuto et al., 2018). TLR dimerization activates several transcription factors, among other the nuclear transcription factor (NF)-KB which induces the production and secretion of inflammatory cytokines, such as tumor necrosis factor (TNF)- $\alpha$ and interleukin (IL)-6, among others.

The NF-KB activation also induces the expression of proteins called Nucleotidebinding domain and leucine-rich repeat-containing pyrin protein 3 (NLRP3) and pro-IL-1 $\beta$. The combination of a TLR activator with an NLRP3 stimulus induce the actiation of the NLRP3 inflammasome, a large oligomer formed by NLRP3, the apoptosis-associated speck-like protein containing a caspase recruitment domain (ASC) proteins and active caspase-1. Active caspase-1 cleaves pro-IL1 $\beta$ and GSDMD to their active pro-inflammatory forms IL-1 $\beta$ and Nt-GDMD. NtGDMD forms pore in the plasma membrane inducing IL-1 $\beta$ secretion and a lytic form of cell death called pyroptosis. (Schroder and Tschopp, 2010; Broz, Pelegrín and Shao, 2019). NLRP3 can be activated by a decrease of $\mathrm{K}^{+}$intracellular 
concentration induced among others by ATP or toxins such as nigericin (Schroder and Tschopp, 2010), or as a consequence of the activation of caspase11 by cytoplasmic LPS (non-canonical inflammasome) (Kayagaki et al., 2011; Hagar et al., 2013; Broz, Pelegrín and Shao, 2019). In the non-canonical inflammasome, active caspase-11 cleaves GSDMD independently of NLRP3, successively, NLRP3 is activated, probably as a consequence of $\mathrm{K}^{+}$efflux, causing IL-1 $\beta$ secretion and amplifying the Nt-GSDMD-induced pyroptosis (Kayagaki et al., 2011; Hagar et al., 2013; Broz, Pelegrín and Shao, 2019).

Ornithine lipids (OLs) are components of bacteria such as Vibrio cholerae, and Pseudomonas aruginosa among others (Vences-Guzmán, Geiger and Sohlenkamp, 2012; Barbosa et al., 2018). OLs are present in low concentrations in the bacteria, but become abundant when bacteria grow in a phosphatedepleted medium, being essential to maintain membrane integrity in those situations where phospholipids cannot be synthesized (Barbosa et al., 2018). The function of OLs is not fully clear yet, it has been suggested to maintain membrane fluidity, protein interaction and antibiotic resistance (Vences-Guzmán, Geiger and Sohlenkamp, 2012; Kim et al., 2018; Córdoba-Castro et al., 2021). Moreover, a very recent study demonstrated the importance of OLs in bacteria pathogenicity (Córdoba-Castro et al., 2021). OLs purified from B. pertussis, A. xylosoxidans and $F$. meningosepticum have been observed to induce IL-6, TNF- $\alpha$ and IL-1 $\beta$ production from macrophages (Kawai and Akagawa, 1989; Okemoto et al., 2008; Palacios-Chaves et al., 2011). However, the bacterial origins of the OL made it difficult to run out of LPS contamination. Moreover these results were in contrast with another work that showed OLs from F. meningosepticum as an inhibitor of LPS-induced immune response in vivo (Kawai et al., 1991).

In this study we aimed to use synthetic OL to investigate the ability of OLs to activate or inhibit the immune response in primary murine and human cells and to identify the receptors involved. We found that OL activates TLR4, prime the NLRP3 and at the same time activates the NLRP3 inflammasome. 


\section{Materials and Methods}

\section{Reagents}

Ultrapure lipopolysaccharide (LPS) from E. coli 0111:B4, $\mathrm{Pam}_{3} \mathrm{CSK}_{4}$, mouse TLR2 neutralizing antibody monoclonal mouse IgG2a (C9A12) and control isotypes mouse lgG2a were from InvivoGen. Mouse TLR4/MD2 complex neutralizing antibody monoclonal rat lgGk clone MTS510 and control isotypes rat IgGk were from eBioscience ${ }^{\mathrm{TM}}$ Invitrogen. FuGENE ${ }^{\circledR}$ was from Promega. MSU crystals from Enzo Life Science. Ornithine lipid was synthesized as described in Supplementary data. Other source of reagents and materials are described in the following sections.

\section{OL liposome preparation}

$\mathrm{CHCl}_{3}$ solutions of $\mathrm{OL} 1 \mathrm{mg} / \mathrm{mL}$ were prepared and lipid films were formed by solvent evaporation under nitrogen stream, dried overnight and kept at $-20^{\circ} \mathrm{C}$. Before each experiment, liposomes were freshly formed by resuspending lipid films into filtered Hepes $10 \mathrm{mM}$ (BioWhittaker) heated at $70{ }^{\circ} \mathrm{C}$, heated 20 minutes at $70^{\circ} \mathrm{C}$ and sonicated for $5 \mathrm{~min}$ in a sonicator bath (Sonitech).

\section{LPS/Fugene ${ }^{\circledR}$ complex preparation for intracellular delivery of LPS}

LPS (InvivoGen) was mixed with the same volume of Fugene ${ }^{\circledR}$ (Promega) in a small volume of Opti-MEM ${ }^{\mathrm{TM}}$ reduced serum medium (Invitrogen) previously heated at $37{ }^{\circ} \mathrm{C}$. The mix was vortexed and incubated 15 minutes at room 
temperature to allow the formation of the complex. Then a volume of Opti-MEM ${ }^{\mathrm{TM}}$ was added to reach a final concentration of $0,5 \%$ Fugene and $1 \mu \mathrm{g} / \mathrm{mL}$ LPS

\section{HEK-Blue $^{\mathrm{TM}}$ cell line culture}

HEK-Blue $^{\mathrm{TM}}$ mTLR4 cells (InvivoGen) were cultured in DMEM F12 (Biowest) supplemented with $10 \%$ heat-inactivated Fetal Bovine Serum Premium (Biowest), 1\% L-glutamine (Lonza), and, from the second passage, $0.1 \mathrm{mg} / \mathrm{mL}$ of Normocin $^{\mathrm{TM}}$ (InvivoGen) and $1 \mathrm{X} \mathrm{HEK}$ Blue $^{\mathrm{TM}}$ selection antibiotic (InvivoGen). Cells were maintained at $37^{\circ} \mathrm{C}$ in a $5 \% \quad \mathrm{CO}_{2}$ atmosphere and tested for mycoplasma contamination on a regular basis with the MycoProbe Mycoplasma Detection Kit following manufacturer instructions (R\&D Systems). To avoid divergence from the parent line, cell cultures were passaged no more than 15 times.

\section{HEK-Blue $^{\mathrm{TM}}$ cell treatments}

HEK-Blue $^{\mathrm{TM}}$ mTLR4 cells (InvivoGen) were seeded at the rate of 70,000 cells and $100 \mu \mathrm{L}$ of culture medium per well in 96-well plates (Greiner Bio-One). After 24 hours, cells were at $80 \%$ confluence and the supernatant was discarded and replaced with $23 \mu \mathrm{L}$ of control solutions (HEPES and water) or stimulants diluted in HEPES and water and $77 \mu \mathrm{L}$ of HEK-Blue ${ }^{\mathrm{TM}}$ Detection medium (InvivoGen) (cell culture medium for real-time detection of SEAP). Following manufacturer's instructions, TLR4-dependent NF-kB activation was measured by the real-time detection of SEAP, through the blue coloration of the HEK-Blue ${ }^{\mathrm{TM}}$ Detection medium. The absorbance at $620 \mathrm{~nm}$ was measured with a BioTek Synergy HT Microplate Readers at $37^{\circ} \mathrm{C}$ after 4,22 and 29 hours of incubation at $37^{\circ} \mathrm{C}$ in a 
$5 \% \mathrm{CO}_{2}$ atmosphere and reported as fold increase with respect to the control condition. Since the highest fold increase induced by both LPS and OL was at 22 hours, we chose to report those data.

\section{Differentiation of bone marrow-derived macrophages (BMDMs)}

Wild-type C57 BL/6J mice were purchased from Harlan. For all experiments, mice between 8-10 weeks of age bred under specific-pathogen-free (SPF) conditions were used in accordance with the Hospital Clínico Universitario Virgen Arrixaca animal experimentation guidelines, and the Spanish national (RD 1201/2005 and Law 32/2007) and EU (86/609/EEC and 2010/63/EU) legislation. According to this legislation, no specific procedure approval is needed when animals are sacrificed to obtain biological material. Bone marrow was obtained from leg bones of mice euthanized by $\mathrm{CO}_{2}$ inhalation and bone marrow-derived macrophages (BMDMs) were obtained. Briefly, bone marrow was flushed out with, resuspended and homogenized in differentiation medium (DMEM (Dulbecco's Modified Eagle's Medium) media, with L-glutamine, w/o sodium pyruvate (BIOWEST) supplemented with $10 \%$ heat-inactivated fetal bovine serum premium (Biowest), $2 \mathrm{mM}$ glutamine (Lonza), $50 \mathrm{U} / \mathrm{mL}$ penicillin, $50 \mu \mathrm{g} / \mathrm{mL}$ streptomycin (PENSTREP, Lonza) and $20 \%$ of supernatant from L929 cultures). The cell suspension generated thereafter was then maintained in petri dishes at $37^{\circ} \mathrm{C}$ in a $5 \% \mathrm{CO}_{2}$ atmosphere. After 2 days more differentiation medium was added and cells maintained for four extra days.

\section{BMDMs treatments}


BMDMs differentiated for six days were washed with Dulbecco's Phosphate buffered saline (PBS) (Thermo Fisher Scientific), PBS was added and the cells were detached from petri dishes, counted, centrifuged $5 \mathrm{~min}$ at $500 \mathrm{~g}$ (Centrifuge 3-18KS, Sigma), resuspended in medium (DMEM medium, with L-glutamine, w/o sodium pyruvate (BIOWEST) supplemented with 10\% heat-inactivated fetal bovine serum premium (Biowest), $2 \mathrm{mM}$ glutamine (Lonza), $50 \mathrm{U} / \mathrm{mL}$ penicillin, $50 \mu \mathrm{g} / \mathrm{mL}$ streptomycin (PEN-STREP, Lonza) to a concentration of $1 \times 10^{6}$ cells/mL and distributed in 96-well plates (100 $\mu \mathrm{L} /$ well), 24-well plates (500 $\mu \mathrm{L} /$ well) or 6-well plates (2 mL/well) (Greiner Bio-One). After 3 hours, 10, 50 or $200 \mu \mathrm{L}$ of medium alone, or supplemented with $\mathrm{Pam}_{3} \mathrm{CSK}_{4} 1 \mu \mathrm{g} / \mathrm{mL}$ or LPS 0.1 or $1 \mu \mathrm{g} / \mathrm{mL}$ (as indicated in the figures) were added. After 4 hours, supernatants were collected, cells washed with PBS and controls and stimulants diluted in Opti$\mathrm{MEM}^{\mathrm{TM}}$ reduced serum medium were added at the rate of $100 \mu \mathrm{L} /$ well $(96-$ well plates), $300 \mu \mathrm{L} /$ well (24-well plate) or $800 \mu \mathrm{L} /$ well (6-well plate) and incubated 18 hours. After incubation, supernatants were collected, centrifuged and assayed for cytokine secretion, while cell lysates were assayed for protein or mRNA expression.

For total incubation with stimulants less than 18 hours, BMDMs differentiated for six days were treated as above and left not 3 but 24 hours in synchronization medium, than medium was discarded and controls, OL, $\mathrm{Pam}_{3} \mathrm{CSK}_{4}$ or LPS diluted in Opti-MEM ${ }^{\mathrm{TM}}$ were added at the rate of $100 \mu \mathrm{L} /$ well (96-well platess), 500 $\mu \mathrm{L} /$ well (24-well plates) or $800 \mu \mathrm{L} /$ well (6-well plates) and incubated 4 hours. After incubation, supernatants were collected, cells washed and a same volume of Opti-MEM ${ }^{\mathrm{TM}}$ or $10 \mu \mathrm{M}$ nigericin (Sigma-Aldrich) was added to LPS or Pam ${ }_{3} \mathrm{CSK}_{4}$ 
treated cells and incubated for 1 hour. Then supernatants were collected centrifuged and assayed for cytokine secretion.

\section{Isolation of Peripheral blood mononuclear cells (PBMCs)}

Whole peripheral blood samples were collected in EDTA anticoagulation tubes from healthy donors. The Institutional Review Board of the Hospital Clínico Universitario Virgen de la Arrixaca approved the use of these blood samples. Informed consent was obtained from all individuals enrolled in the study following the principles set out in the WMA Declaration of Helsinki and samples were stored in the Biobanco en Red de la Región de Murcia (PT13/0010/0018) integrated in the Spanish National Biobanks Network (B.000859). PBMCs were obtained by Ficoll gradient centrifugation in Histopaque-1077 (Sigma-Aldrich) using SepMate $^{\mathrm{TM}}$ isolation tubes from STEMCELL ${ }^{\mathrm{TM}}$ following manufacturer's instructions. Isolated PBMCs were resuspended in RPMI 1640 (Sigma-Aldrich) supplemented with $10 \%$ heat-inactivated FBS premium (Biowest) and maintained at $37^{\circ} \mathrm{C}$ in a $5 \% \mathrm{CO}_{2}$ atmosphere.

\section{PBMCs treatments}

A suspension of freshly isolated PBMCs were distributed in round bottom 96-well plates (50 $\mu \mathrm{L}, 100,000$ cells per well) and $50 \mu \mathrm{L}$ of RPMI 1640 with control solutions (HEPES and water) or stimulants were added and cells maintained at $37^{\circ} \mathrm{C}$ in a $5 \% \mathrm{CO}_{2}$ atmosphere for 4 or 18 hours. Then supernatants were collected, centrifuged and assayed for cytokine secretion.

\section{Cytokine assays}


Murine TNF- $\alpha$ and IL-1 $\beta$ were quantified in cell supernatants using ELISA ${ }^{\text {TM }}$ Kit from Invitrogen and human IL-1 $\beta$ using Instant ELISA ${ }^{\text {TM }}$ Kit from Invitrogen, following manufacturer's instructions. Absorbance was read with a BioTek Synergy HT Microplate Reader. All other cytokines were quantified in cell supernatants using a custom Multiplex Kit procartaplex from Thermo Fisher Scientific, following manufacturer's instructions using a Luminex MAGPIX System - Millipore.

\section{LDH assay}

LDH activity was quantified in cell lysate of untreated cells and in all cell supernatants using the Cytotoxicity Detection Kit (LDH) from Merck, following manufacturer's instructions. The absorbance at 492 and $620 \mathrm{~nm}$ was measured with a BioTek Synergy HT Microplate Reader every minute for $20 \mathrm{~min}$. The slopes of the increase in absorbance with respect to the time were calculated, background value was subtracted and the value of each supernatant was reported as percentage of the sum of the value measured in the supernatant and the lysate of the untreated condition (total LDH).

\section{Cell lysis}

For Western blot and LDH assay, cells were lysed with $2 \%$ Triton lysis buffer composed of $150 \mathrm{mM} \mathrm{NaCl}$ (Immobilon), 2\% Triton X-100 (Sigma-Aldrich), 50 $\mathrm{mM}$ Tris- $\mathrm{HCl} \mathrm{pH}=8$ (Sigma-Aldrich) supplemented with $100 \mu \mathrm{L} / \mathrm{mL}$ of protease inhibitor(Sigma-Aldrich). Cells were scraped in cold lysis buffer on ice, lysates were incubated 30 minutes in ice with a vortex every 10 minutes and then 
centrifuged 10 minutes at 13,000 $\mathrm{g}$ in a microcentrifuge (1-14K, Sigma) to remove pellet containing cell debris.

\section{Western blot}

Lysates from cells treated in 6-well plate were heated for 5 minutes at $100^{\circ} \mathrm{C}$ with the same volume of Leammli Buffer 2X (Sigma-Aldrich). Samples were separated in a vertical electrophoresis on $12 \%$ polyacrylamide gels (Biorad) and transferred for $1 \mathrm{~h}$ at $350 \mathrm{~mA}$ to nitrocellulose membranes (BioRad). The membranes were probed with primary antibodies anti-GSDMD rabbit monoclonal (Abcam ab209845 1:5000), anti-casp-11 rabbit monoclonal (Abcam ab180673 1:1000), anti-IL-1 $\beta$ rabbit polyclonal (sc-7884 Santa Cruz 1:1000), anti-NLRP3 mouse monoclonal (Adipogen AG-20B-0014 1:1000) or horseradish peroxidase (HRP)conjugated anti- $\beta$-actin (sc-47778 Santa Cruz 1:25000). The blots were developed using appropriate HRP-conjugated secondary antibody from GE Healthcare (1:5000) and an enhanced chemiluminescence (ECL) reagents (GE Healthcare), according to the manufacturer's protocol, in a Chemidoc equipment (BioRad).

\section{Quantitative PCR}

BMDMs cells treated in 6-well plate where washed twice with PBS before total RNA purification using the RNeasy kit (Qiagen) following manufacturer's recommendations and quantified on a nanodrop 2000 (Thermo Fisher). Reverse transcription was realized using iScriptTM cDNA Synthesis kit (BioRad) following manufacturer's instructions. Quantitative PCR (qPCR) was performed in an iQTM 5 Real Time PCR detection System (BioRad) with a SYBR Green mix (Takara) 
and predesigned primers obtained from Sigma-Aldrich (KiCqStart® Primers). The presented relative gene expression levels were calculated using the $2^{-\triangle \Delta C T}$ method (Livak and Schmittgen, 2001) normalizing to GAPDH expression as endogenous controls.

\section{Endotoxin test}

LPS was quantified in OL liposomes using the Pierce ${ }^{\mathrm{TM}}$ Chromogenic Endotoxin Quant Kit (Thermo Fischer) following the manufacturer's instructions.

\section{Statistical analysis}

Each dot in the graphics represents the mean value of biological triplicates from an independent experiment. Each bar represents the mean value and error bars the standard deviation from the number of independent experiments indicated in the figure. For two-group comparisons, a two-tailed unpaired, paired or ratio $t$-test was used, comparisons of multiple groups were analyzed by one-way analysis of variance ANOVA with Bonferroni's multiple-comparison test using Prism software (Graph-Pad Software, Inc.). $p$ value is indicated as ${ }^{*} p<0.05 ; p>0.05$ not significant (ns or not indicated). 


\section{Results}

\section{OL activates TLR4 and prime macrophages for NLRP3 activation}

We tested the pro-inflammatory proprieties of synthetic OL by incubating 4 or 18 hours BMDMs and human PBMCs with increasing amounts of OL or LPS (as positive control of TLR activation) or 4 hours with LPS followed by 1 hour of nigericin (as positive control of canonical NLRP3 activation). We observed that $\mathrm{OL}$ induced the secretion of the pro-inflammatory cytokines TNF- $\alpha$ and IL-1 $\beta$ in both cell types (Fig. 1 A-D). Multiplex assay carried out with supernatants of BMDMs treated with OL also revealed a significant increase in the secretion of IL-18, IL-6, CXCL10, IL-2 and IL-10 (Fig. 1 E-I), but not in IL-1 $\alpha$ secretion respect to the untreated controls (Fig. S1).

Since the secretion of TNF- $\alpha$, IL-6, CXCL10, IL-2 and IL-10 induced by OL is typical of TLR activation (Fitzgerald and Kagan, 2020) and we previously observed that synthetic ionizable lipids activate TLR2 and TLR4 (Pizzuto et al., 2016, 2018), we wondered whether OL was able to activate those receptors. We therefore tested the ability of OL to induce TNF- $\alpha$ secretion in BMDMs preincubated with antibodies blocking TLR4 or TLR2. As shown in figure 2, blocking TLR2 had no effect on the OL-induced TNF-a secretion (Fig. 2 B), which was instead dependent on TLR4 (Fig. $2 \mathrm{~A}$ and C). The antibodies blocking TLR2 were able to prevent TNF- $\alpha$ secretion induced by the TLR2 activator $\mathrm{Pam}_{3} \mathrm{CSK}_{4}$ (Fig. $2 \mathrm{D})$, demonstrating that the antibodies were effective at the concentration used and that the TNF- $\alpha$ secretion induced in BMDMs by OL is solely due to TLR4 activation. To confirm the ability of OL to activate TLR4, we used the TLR4 reporter cell line HEKBlue-mTLR4. By treating the cells with increasing amounts of OL, we observed that, similarly to LPS treatment, OL was able to induce TLR4dependent NF-kB activation in the reporter cells. Endotoxin tests verified that the OL preparations used were free of LPS contamination (Fig. S2).

\section{OL activates the NLRP3 inflammasome}

The secretion of $\mathrm{IL}-1 \beta$ and $\mathrm{IL}-18$ is an indicator of the activation of an inflammasome. Among the inflammasomes, NLRP3 inflammasome is known to be activated by particulate matter, nanoparticles and liposomes made of synthetic ionizable lipids (Lonez et al., 2014; He, Hara and Núñez, 2016; Pizzuto et al., 2018). Therefore we tested whether OL was able to activate the NLRP3 
inflammasome. We found that OLs was inducing the expression of NIrp3 and $/ / 1 b$, priming the cells for NLRP3 activation (Fig. $3 \mathrm{~A}-\mathrm{C}$ ). Also proteins involved in both canonical and non-canonical NLRP3 inflammasome activation such as caspase11 and GSDMD were upregulated by OL (Fig. $3 \mathrm{C}$ ).

In order to determine whether OL was also able to activate the NLRP3 inflammasome, we compared IL-1 $\beta$ secretion induced by OL in the absence and in the presence of NLRP3 inhibitor MCC950, the caspase-1 inhibitor VX-765 or a high $\mathrm{K}^{+}$buffer and in BMDM deficient of NLRP3 $\left(\mathrm{NIrp3}^{-/-}\right)$, ASC $\left(\right.$Pycard $\left.^{-I^{-}}\right)$and caspase-1/11 (Casp1/11 $\left.1^{--}\right)$. OL was unable to induce IL-1 $\beta$ secretion in BMDM phenotype missing NLRP3 inflammasome components (Fig. 4 A) and the secretion in wild type macrophages was abrogated by inhibitors of the pathway at NLRP3 and caspase-1 level, demonstrating that OL activates the NLRP3 inflammasome in a $\mathrm{K}^{+}$efflux dependent manner (Fig. 4 B). By measuring TNF- $\alpha$ in BMDM from wild type and ASC deficient mice $\left(\right.$ Pycard $\left.^{--}\right)$we assessed that the ability of OLs to activate TLR4 was independent of inflammasome activation (Fig. S3). To investigate the ability of OL to activate human NLRP3, we incubated PBMCs 18 hours with OL in the absence or in the presence of NLRP3 inhibitor MCC950. The OL-induced IL-1 $\beta$ secretion was abrogated by the inhibitor, demonstrating that OL activates NLRP3 inflammasome in human cells.

We observed that OL is able to prime macrophages for NLRP3 activation and

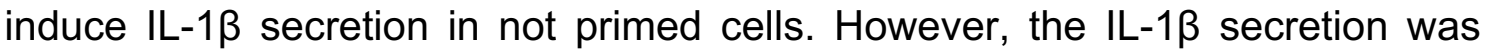
much lower than the one induced by nigericin in cells primed with LPS (Fig. 1C). This might be due to the fact that cells primed with LPS presented an enhanced NLRP3 and pro-IL-1 $\beta$ expression (Fig. 3B). To properly compare OL with other NLRP3 stimuli, we primed the cells with LPS or Pam ${ }_{3} \mathrm{CSK}_{4}$, and then incubated with OL, nigericin (canonical NLRP3 activator) or LPS delivered intracellularly by Fugene $®$ transfection agent (non-canonical NLRP3 activator). Surprisingly, $\mathrm{Pam}_{3} \mathrm{CSK}_{4}$ but not LPS priming strongly increased the amount of NLRP3dependent IL-1 $\beta$ secretion induced by OL (Fig. $5 \mathrm{~A}$ and $\mathrm{B}$ ), although it remained lower than the one induced by nigericin or intracellular LPS. Moreover, caspase1/11-independent TNF- $\alpha$ secretion induced by OL and LPS was as well enhanced by $\mathrm{Pam}_{3} \mathrm{CSK}_{4}$ but not by LPS priming (Fig. S4). Also intracellular LPSdependent but not nigericin-dependent IL-1 $\beta$ secretion was enhanced in 
$\mathrm{Pam}_{3} \mathrm{CSK}_{4}$ primed cells (Fig. 5), by contrast, no change were observed for pyroptosis (LDH release) induced by all compounds (Fig. S5).

\section{OL activates caspase-11 and the non-canonical NLRP3 inflammasome}

Since the effect of $\mathrm{Pam}_{3} \mathrm{CSK}_{4}$ priming over OL was similar to the one observed for intracellularly delivered LPS (Fig. 5, S4), we wondered whether OL, like intracellular LPS activates NLRP3 inflammasome through the non-canonical pathway, which involves the activation of caspase-11 and NLRP3-independent GSDMD cleavage and LDH release as first steps (Kayagaki et al., 2013). We incubated $\mathrm{Pam}_{3} \mathrm{CSK}_{4}$ primed BMDMs derived from wild type, NIrp3 ${ }^{-/-}$and Casp1/11//- mice for 18 hours with OL or intracellular LPS or 1 hour with nigericin and then quantified GSDMD cleavage in lysates. We found that OL was able to induce GSDMD cleavage in both wild type and NLRP3-deficient BMDMs but not in BMDMs deficient of both caspase-1/-11 (Fig. 6). This indicates that NLRP3 is not necessary for GSDMD cleavage induced by OL and strongly suggests that OL activates caspase-11 and the non-canonical NLRP3 inflammasome.

\section{OL is a partial agonist of TLR4 and caspase-11}

Compared to LPS, OL appeared to be a weaker activator of TLR4 and caspase11 (Fig. 1D, 2E, 3C, 5 and S4). This suggest that OL is a partial TLR4 and/or caspase-11 agonist. Indeed, partial agonists weakly activate their receptors when administered alone but inhibit the activation of those same receptors by the full agonist (Kenakin, 2017). To investigate this hypothesis, we tested the ability of OLs to decrease intracellular LPS-induced TNF- $\alpha$ and IL-1 $\beta$ secretion by coincubating BMDMs with OL and LPS or LPS complexed with Fugene ${ }^{\circledR}$ transfection agent. As shown in figure 7, the same concentration and preparation of OLs that induced TNF- $\alpha$ and IL-1 $\beta$ secretion in BMDMs was able to inhibit TNF- $\alpha$ and IL-1 $\beta$ release induced by LPS. OL was not able to inhibit TNF- $\alpha$ induced by $\mathrm{Pam}_{3} \mathrm{CSK}_{4}$, nor the secretion of $\mathrm{IL}-1 \beta$ induced by nigericin, demonstrating a specific action towards LPS receptors and strongly suggesting that OLs is a TLR4 and caspase-11 partial agonist. 


\section{Discussion}

Previous studies reported on the ability of bacteria purified OLs to induce IL-6, TNF- $\alpha$ and IL-1 $\beta$ secretion (Kawai and Akagawa, 1989; Okemoto et al., 2008). Other studies claimed that OLs are either inert or inhibitors of LPS-induced inflammatory reactions (Kawai et al., 1991; Palacios-Chaves et al., 2011). This may be due to differences in the lipid origin, preparation or purification process, experimental differences as well as the bacterial origin make difficult to interpretate those data properly. By using synthetic OL, we demonstrated that OL inhibits the activation of TLR4 and non-canonical NLRP3 inflammasome by LPS. However, in the absence of LPS, the same OL preparation activates those same receptors, inducing a large spectrum of pro-inflammatory cytokines secretion in both human blood mononuclear cells and murine macrophages. This indicates that OL is a partial agonist of TLR4 and, probably also of caspase-11. Indeed a partial agonist weakly activates a receptor when administered alone but inhibits the activation of the receptor by the full agonist (Kenakin, 2017).

The cytokine secretion induced by OL was weak but strongly enhanced by priming the cells with TLR2 activator $\mathrm{Pam}_{3} \mathrm{CSK}_{4}$. Moreover, the synthetic OL tested in the present study is saturated, with a chain length of 14 carbon atoms $(C 14: 0)$. It is possible that other chain length found in bacteria have a stronger or weaker TLR4 and NLRP3 inflammasome activity. C16:0 OL from B. pertussis has been observed to induce TNF- $\alpha$ and IL-6 secretion, while C18:0 OL from $B$. abortus was inactive (Palacios-Chaves et al., 2011), being this in agreement with our previous studies indicating that C18-ionizable lipids do not activate TLR4 (Pizzuto et al., 2016, 2018).

The activation of TLR4 by C14:0 OL was demonstrated in this study by the activation of NF-kB in TLR4 reporter cell line as well as by the strong reduction of OL-induced TNF- $\alpha$ in the presence of antibodies blocking TLR4. As expected for TLR4 activation, OL induced the expression of NLRP3, pro-IL-1 $\beta$, GSDMD and caspase-11 proteins, priming the macrophages for inflammasome activation. Without the need of a second signal, OL also induced NLRP3 activation probably dependent on caspase-11. In the canonical pathway GSDMD is cleaved by caspase-1 only after NLRP3 activation, whereas in the non-canonical pathway GSDMD is cleaved by caspase-11 upstream of NLRP3 (Kayagaki et al., 2011, 2013; Hagar et al., 2013), as occurs in macrophages treated with OL. In support 
of the activation of caspase-11, OL-induced IL-1 $\beta$ secretion was shown to be enhanced by IFN- $\gamma$ (Kawai and Akagawa, 1989), a known booster of the caspase11 and non-canonical NLRP3 inflammasome pathway (Brubaker et al., 2020).

Also in human PBMCs OL induced an NLRP3 activation without the need of a second signal, indeed the IL-1 $\beta$ secretion induced by OL was blocked by the NLRP3 inhibitor MCC950 (Coll et al., 2015). However, it must be noted that in PBMCs also extracellular LPS induced IL-1 $\beta$ secretion. This is in line with previous studies showing that the activation of TLR4 is sufficient to activate NLRP3, through the alternative NLRP3 pathway in PBMCs (Gaidt et al., 2016). PBMCs also respond to canonical NLRP3 activators (Gaidt et al. , 2016), therefore the activation of human NLRP3 by OL might be both dependent or independent of TLR4. It must be noted that caspase-11 activation by LPS requires the delivery of LPS into the cytosol (Kayagaki et al., 2013). OL form liposomes and due to its ionizable nature, it is likely that those liposomes will reach the intracellular space as observed for ionizable synthetic lipids (Lonez, Vandenbranden and Ruysschaert, 2008; Pizzuto et al., 2018). In support of OL entering the cells as liposomes, liposomes formation is indispensable for OL-induced IL-1 $\beta$ secretion (Okemoto et al., 2008).

The ability of OL to both prime and activate NLRP3 inflammasome have been also observed for few other compounds like hyaluronan, biglycan and heme among others (Babelova et al., 2009; Yamasaki et al., 2009; Erdei et al., 2018) and for lipopolyamines, synthetic ionizable lipids not present in nature and used to transport proteins and transfect nucleic acids (Lonez et al., 2014; Pizzuto et al., 2018). NLRP3 and TLR4 activation stimulates the adaptative immune response and is required for successful vaccine formulation (Awate, Babiuk and Mutwiri, 2013; Pulendran, S. Arunachalam and O'Hagan, 2021). Therefore, triggers of the NLRP3 pathway like Alum are used as adjuvants in vaccine formulations together with a TLR4 activator, the antigen and the delivery system (Eisenbarth et al., 2008; Pulendran, S. Arunachalam and O'Hagan, 2021). The fact that lipopolyamines are delivery system with the intrinsic ability to activate TLRs and NLRP3 resulted in effective adjuvant-free vaccination of mice treated with the antigen simply mixed with lipopolyamine (Pizzuto et al., 2018). OL has a structure similar to lipopolyamine, therefore its ability to transport antigens or 
deliver nucleic acids might be explored in order to propose its use in adjuvantfree vaccine.

\section{Conclusions}

OL is able to fully replace LPS pathogenicity by activating both TLR4 and noncanonical NLRP3 inflammasome. The consequent secretion of TNF- $\alpha$ and IL-1 $\beta$ is much weaker than LPS, but is strongly enhanced by priming the cells with TLR2 activators. OL might be used by the bacteria as a substitute of LPS to keep pathogenicity in the absence of LPS. By contrast, as a partial agonist OL is able to inhibit LPS-induced inflammatory cytokine secretion suggesting a role on bacterial escape strategy.

\section{Competing interests}

LH-N and PP are co-founders of Viva in vitro diagnostics SL, but declare that the research was conducted in the absence of any commercial or financial relationships that could be construed as a potential conflict of interest. The other authors declare no competing interests.

\section{Funding}

This work was supported by grants to PP from MCIN/AEI/10.13039/501100011033 (grant PID2020-116709RB-I00), Fundación Séneca (grants 20859/PI/18, 21081/PDC/19 and 0003/COVI/20) and European Research Council (grants ERC-2013-CoG 614578 and ERC-2019-PoC 899636). L.H-N. was supported by the fellowship 21214/FPI/19 (Fundación Séneca, Región de Murcia, Spain) and CM-L by was funded by the fellowship PRE2018087063 (Ministerio economía y competitividad). MP was supported by a Fond National de la Recherche Scientifique postdoctoral fellowship (CR 32774874) and Juan de la Cierva-Formación postdoctoral fellowship (FJC2018-036217-I). 


\section{Authors' Contributions}

MP, LH-N, CM-L and SR-L experimental execution; MP, LH-N, CM-L, SR-L, J-

MR, and PP data analysis; MP and PP conceived the experiments; MP prepared the figures and wrote the paper; J-MR and PP draft revision, provided funding and overall supervision of the study.

\section{Acknowledgments}

We thanks M.C. Baños and A.I. Gomez (IMIB, Murcia, Spain) for technical assistance with cell culture. We would like to thank all the members of Dr. Pelegrin's laboratory for comments and suggestions.

\section{References}

Awate, S., Babiuk, L.A. and Mutwiri, G. (2013) "Mechanisms of action of adjuvants," Frontiers in Immunology, 4(MAY), p. 114. doi:10.3389/FIMMU.2013.00114/BIBTEX.

Babelova, A. et al. (2009) "Biglycan, a danger signal that activates the NLRP3 inflammasome via toll-like and P2X receptors," The Journal of biological chemistry, 284(36), pp. 24035-24048. doi:10.1074/JBC.M109.014266.

Barbosa, L.C. et al. (2018) "Accumulation of ornithine lipids in Vibrio cholerae under phosphate deprivation is dependent on VC0489 (OlsF) and PhoBR system," Microbiology (United Kingdom), 164(3), pp. 395-399. doi:10.1099/mic.0.000607.

Barton, G.M. and Kagan, J.C. (2009) "A cell biological view of $\{$ Toll\}-like receptor function: regulation through compartmentalization," Nat. Rev. Immunol, 9, pp. 535-542.

Broz, P., Pelegrín, P. and Shao, F. (2019) "The gasdermins, a protein family executing cell death and inflammation," Nature Reviews Immunology [Preprint]. Nature Publishing Group. doi:10.1038/s41577-019-0228-2. 
Brubaker, S.W. et al. (2020) "A Rapid Caspase-11 Response Induced by IFNy Priming Is Independent of Guanylate Binding Proteins," iScience, 23(10). doi:10.1016/J.ISCI.2020.101612.

Coll, R.C. et al. (2015) "A small-molecule inhibitor of the NLRP3 inflammasome for the treatment of inflammatory diseases," Nature Medicine 2015 21:3, 21(3), pp. 248-255. doi:10.1038/nm.3806.

Córdoba-Castro, L.A. et al. (2021) "Ornithine Lipids in Burkholderia spp. Pathogenicity," Frontiers in Molecular Biosciences, 7, p. 457. doi:10.3389/fmolb.2020.610932.

Eisenbarth, S.C. et al. (2008) "Crucial role for the Nalp3 inflammasome in the immunostimulatory properties of aluminium adjuvants," Nature 2008 453:7198, 453(7198), pp. 1122-1126. doi:10.1038/nature06939.

Erdei, J. et al. (2018) "Induction of NLRP3 Inflammasome Activation by Heme in Human Endothelial Cells," Oxidative medicine and cellular longevity, 2018. doi:10.1155/2018/4310816.

Fitzgerald, K.A. and Kagan, J.C. (2020) "Toll-like Receptors and the Control of Immunity," Cell. Cell Press, pp. 1044-1066. doi:10.1016/j.cell.2020.02.041.

Gaidt, M.M. et al. (2016) "Human Monocytes Engage an Alternative Inflammasome Pathway," Immunity, 44(4), pp. 833-846. doi:10.1016/j.immuni.2016.01.012.

Gay, N.J. et al. (2014) "Assembly and localization of Toll-like receptor signalling complexes," Nature Reviews Immunology, pp. 546-558. doi:10.1038/nri3713.

Hagar, J.A. et al. (2013) "Cytoplasmic LPS Activates Caspase-11: Implications in TLR4-Independent Endotoxic Shock," Science, 341(6151), pp. 12501253. doi:10.1126/science. 1240988.

He, Y., Hara, H. and Núñez, G. (2016) "Mechanism and Regulation of NLRP3 Inflammasome Activation," Trends in Biochemical Sciences. Elsevier Ltd, pp. 1012-1021. doi:10.1016/j.tibs.2016.09.002. 
Kawai, Y. et al. (1991) "Protection of mice from lethal endotoxemia by use of an ornithine-containing lipid or a serine-containing lipid," Infection and Immunity, 59(8), pp. 2560-2566. doi:10.1128/iai.59.8.2560-2566.1991.

Kawai, Y. and Akagawa, K. (1989) "Macrophage activation by an ornithinecontaining lipid or a serine-containing lipid," Infection and Immunity, 57(7), pp. 2086-2091. doi:10.1128/iai.57.7.2086-2091.1989.

Kayagaki, N. et al. (2011) "Non-canonical inflammasome activation targets caspase-11," Nature, 479(7371), pp. 117-121. doi:10.1038/nature10558.

Kayagaki, N. et al. (2013) "Noncanonical inflammasome activation by intracellular LPS independent of TLR4," Science, 341(6151), pp. 12461249. doi:10.1126/science. 1240248.

Kenakin, T.P. (2017) "Drug Antagonism: Orthosteric Drug Effects," Pharmacology in Drug Discovery and Development, pp. 65-100. doi:10.1016/B978-0-12-803752-2.00004-1.

Kim, S.K. et al. (2018) "Bacterial ornithine lipid, a surrogate membrane lipid under phosphate-limiting conditions, plays important roles in bacterial persistence and interaction with host," Environmental Microbiology, 20(11), pp. 3992-4008. doi:10.1111/1462-2920.14430.

Livak, K.J. and Schmittgen, T.D. (2001) "Analysis of relative gene expression data using real-time quantitative PCR and the 2(-Delta Delta $\mathrm{C}(\mathrm{T})$ ) Method," Methods (San Diego, Calif.), 25(4), pp. 402-408. doi:10.1006/METH.2001.1262.

Lonez, C. et al. (2014) "Cationic lipid nanocarriers activate Toll-like receptor 2 and NLRP3 inflammasome pathways," Nanomedicine: Nanotechnology, Biology, and Medicine, 10(4), pp. 775-782.

Lonez, C. et al. (2015) "Critical residues involved in Toll-like receptor 4 activation by cationic lipid nanocarriers are not located at the lipopolysaccharide-binding interface," Cellular and Molecular Life Sciences, 72(20), pp. 3971-3982. doi:10.1007/s00018-015-1915-1.

Lonez, C., Vandenbranden, M. and Ruysschaert, J.-M. (2008) "Cationic liposomal lipids: from gene carriers to cell signaling," Prog. Lipid Res., 47(5), pp. 340-347. doi:10.1016/j.plipres.2008.03.002. 
Okemoto, K. et al. (2008) "The preparation of a lipidic endotoxin affects its biological activities," Biological and Pharmaceutical Bulletin, 31(10), pp. 1952-1954. doi:10.1248/bpb.31.1952.

Palacios-Chaves, L. et al. (2011) "Brucella abortus ornithine lipids are dispensable outer membrane components devoid of a marked pathogenassociated molecular pattern," PloS one, 6(1). doi:10.1371/JOURNAL.PONE.0016030.

Pizzuto, M. et al. (2016) "Toll-like receptor 2 promiscuity is responsible for the immunostimulatory activity of nucleic acid nanocarriers," Journal of Controlled Release: Official Journal of the Controlled Release Society, 247, pp. 182-193. doi:10.1016/j.jconrel.2016.12.029.

Pizzuto, M. et al. (2018) "Cationic lipids as one-component vaccine adjuvants: A promising alternative to alum," Journal of Controlled Release, 287, pp. 67-77. doi:10.1016/j.jconrel.2018.08.020.

Pizzuto, M. et al. (2019) "Saturation of acyl chains converts cardiolipin from an antagonist to an activator of Toll-like receptor-4," Cellular and Molecular Life Sciences, 76(18), pp. 1-12. doi:10.1007/s00018-019-03113-5.

Pulendran, B., S. Arunachalam, P. and O'Hagan, D.T. (2021) "Emerging concepts in the science of vaccine adjuvants," Nature Reviews Drug Discovery 2021 20:6, 20(6), pp. 454-475. doi:10.1038/s41573-02100163-y.

Raghavan, B. et al. (2012) "Metal allergens nickel and cobalt facilitate TLR4 homodimerization independently of MD2," EMBO Reports, 13(12), pp. 1109-1115. doi:10.1038/embor.2012.155.

Rathinam, V.A.K. et al. (2012) "TRIF licenses caspase-11-dependent NLRP3 inflammasome activation by gram-negative bacteria.," Cell, 150(3), pp. 606-19. doi:10.1016/j.cell.2012.07.007.

Schmidt, M. et al. (2010) "Crucial role for human Toll-like receptor 4 in the development of contact allergy to nickel," Nature Immunology, 11(9), pp. 814-819. doi:10.1038/ni.1919. 
Schroder, K. and Tschopp, J. (2010) "The Inflammasomes," Cell, 140(6), pp. 821-832. doi:10.1016/J.CELL.2010.01.040/ATTACHMENT/DDC1AD197AB1-43F5-B1AF-F7104761B11C/MMC1.PDF.

Uno, K. et al. (2007) "Toll-like receptor (TLR) 2 induced through TLR4 signaling initiated by Helicobacter pylori cooperatively amplifies iNOS induction in gastric epithelial cells," American Journal of Physiology - Gastrointestinal and Liver Physiology, 293(5), pp. 1004-1012. doi:10.1152/AJPGI.00096.2007/ASSET/IMAGES/LARGE/ZH301107491 50009.JPEG.

Vences-Guzmán, M.Á., Geiger, O. and Sohlenkamp, C. (2012) "Ornithine lipids and their structural modifications: From $\mathrm{A}$ to $\mathrm{E}$ and beyond," FEMS Microbiology Letters, 335(1), pp. 1-10. doi:10.1111/j.15746968.2012.02623.x.

Yamasaki, K. et al. (2009) "NLRP3/cryopyrin is necessary for interleukin-1beta (IL-1beta) release in response to hyaluronan, an endogenous trigger of inflammation in response to injury," The Journal of biological chemistry, 284(19), pp. 12762-12771. doi:10.1074/JBC.M806084200. 


\section{Figures and legends}

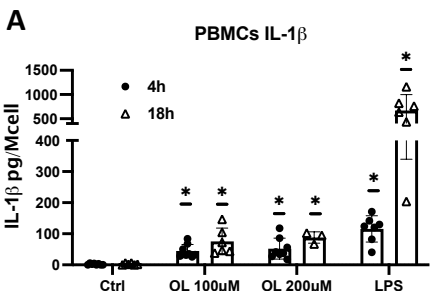

B
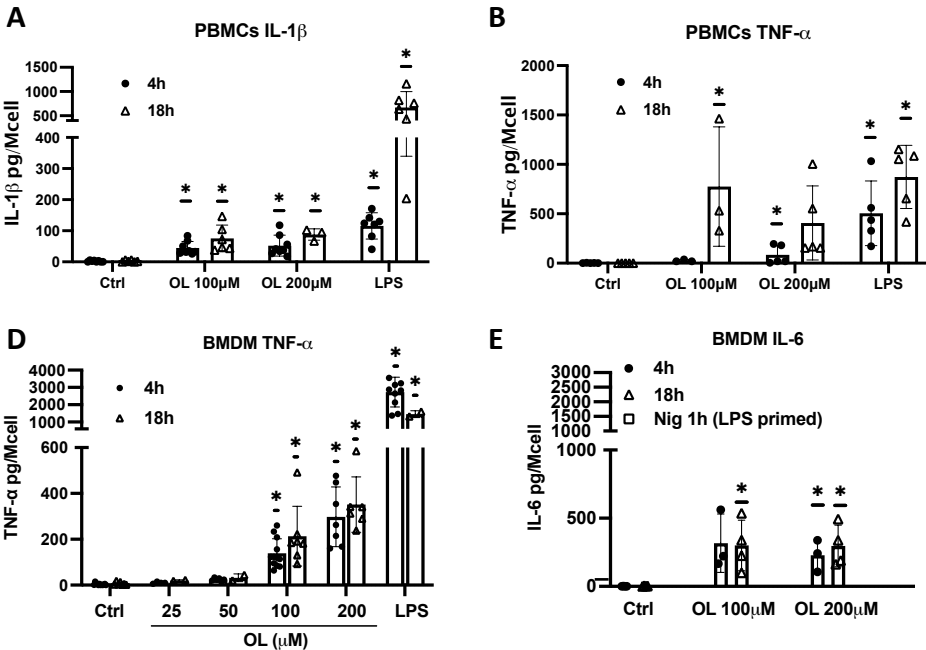

$\mathbf{E}$
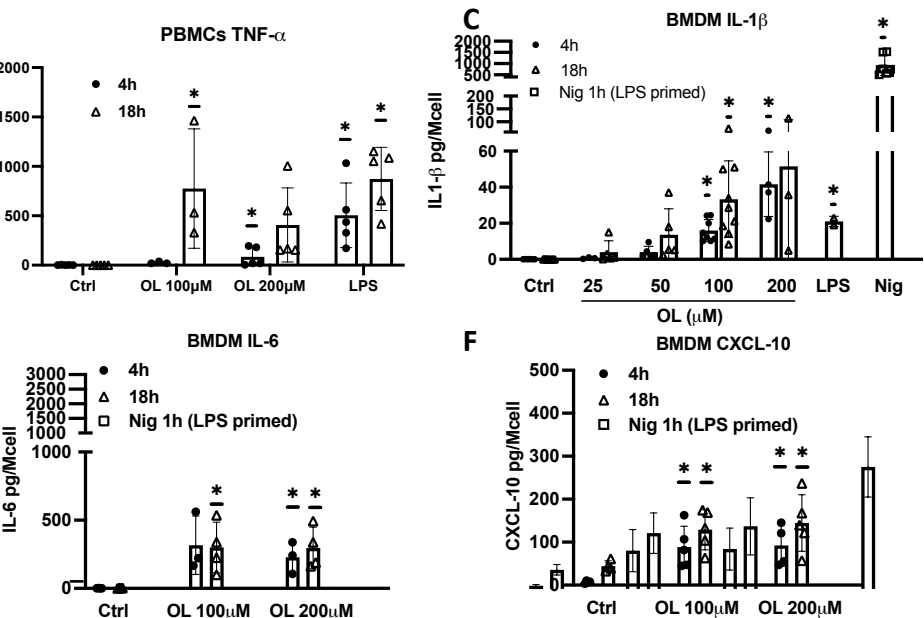

$\mathbf{F}$

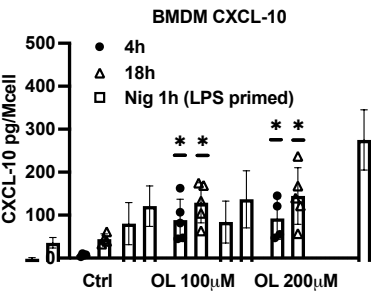

G

H
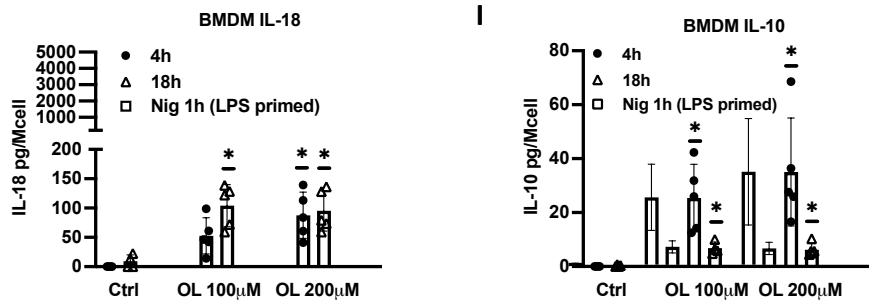

Figure 1: OL induces the secretion of pro-inflammatory cytokines. A,B) Human peripheral blood derived mononuclear cells (PBMCs) from healthy donors were incubated for 4 or $18 \mathrm{~h}$ without (Ctrl) or with LPS (100 ng/mL) or OL (100 and $200 \mu \mathrm{M})$. IL-1 $\beta$ (A) and TNF- $\alpha$ (B) were quantified in supernatants by ELISA. C-I) Bone marrow derived macrophages (BMDM) from wild type mice were incubated for 4 or $18 \mathrm{~h}$ without (Ctrl), or with LPS $(100 \mathrm{ng} / \mathrm{mL})$ or $\mathrm{OL}(25,50,100$ or $200 \mu \mathrm{M})$, in some wells after $4 \mathrm{~h}$ incubation with LPS $(100 \mathrm{ng} / \mathrm{mL})$ the cells were washed and incubated for $1 \mathrm{~h}$ with nigericin $(10 \mu \mathrm{M}, \mathrm{Nig})$. IL-1 $\beta$ (C) and TNF- $\alpha(\mathrm{D})$ were quantified in supernatants by ELISA. IL-6 (E), CXCL10 (F), IL-2 (G), IL-18 (H) and IL10 (I) were quantified by luminex assay.

Bars represent the mean of three or more experiments represented by different symbols ( $N=3$ to 5 ). Statistical analysis: one-way ANOVA for OL, unpaired $t$-test for LPS and Nig. Significant difference with respect to the control condition for $p<0.05\left(^{*}\right)$. 

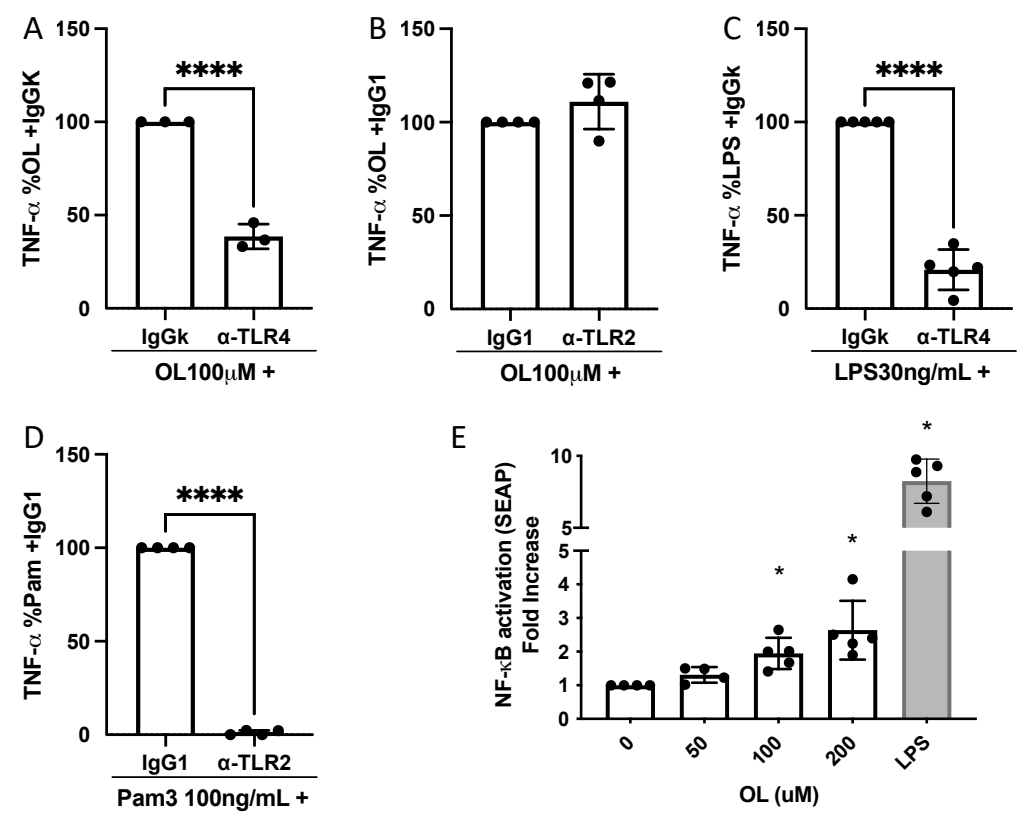

Figure 2: OL activates TLR4. A-D) Bone marrow derived macrophages (BMDM) from wild type mice were incubated for $18 \mathrm{~h}$ without (Ctrl), or with LPS (30 ng/ml), Pam3CSK $(100 \mathrm{ng} / \mathrm{mL})$ or OL $(100 \mu \mathrm{M})$ in the presence of $5 \mu \mathrm{g} / \mathrm{mL}$ of antibodies blocking TLR4 or TLR2 or their respective isotype control antibodies. TNF- $\alpha$ was quantified in collected supernatants by ELISA and reported here as percentage of the secretion measured in the presence of the isotype control antibody. E) HEKBlue-mTLR4 were incubated for $22 \mathrm{~h}$ without $(0)$, or with OL $(50,100,200 \mu \mathrm{M})$ or LPS (100 ng/mL, grey bar). TLR4-dependent NF-kB activation was quantified by Secreted alkaline phosphatase (SEAP) assay and reported here as fold increase with respect to the control condition.

Bars represent the mean of three or more experiments represented by dots ( $N=3$ to 5 ). Statistical analysis: A-D Paired $t$-test, E) one-way ANOVA A-E) significant difference with respect to the control condition for $p<0.05\left(^{*}\right)$.
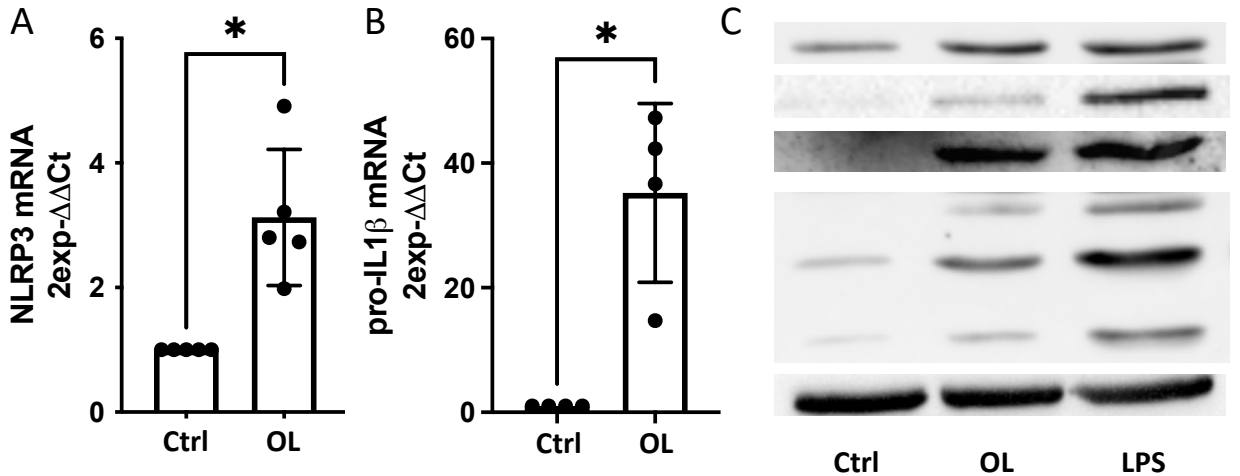

GSDMD NLRP3

Pro-IL1 $\beta$

Unspecific band

Casp11 p43

Casp11 p36

B-Actin

Figure 3: OL primes macrophages for inflammasome activation. (A,B) NLRP3 (A) and IL1 $\beta$ (B) mRNA expression from bone marrow derived macrophages (BMDM) from wild type mice were incubated for $18 \mathrm{~h}$ without (Opt) or with OL $(100 \mu \mathrm{M})$ or LPS $(100 \mathrm{ng} / \mathrm{mL})$. Bars represent the mean of four experiments represented by dots $(\mathrm{N}=4)$. Statistical analysis: Unpaired $t$-test, significant difference with respect to the control condition for $p<0,05\left(^{*}\right)$ C) GSDMD, NLRP3, pro-IL-1 $\beta$ and Casp-11 protein levels were quantified by Western Blot from cells treated as in (A). $\beta$-actin was used as charge control. Data are representative of three independent experiments. 

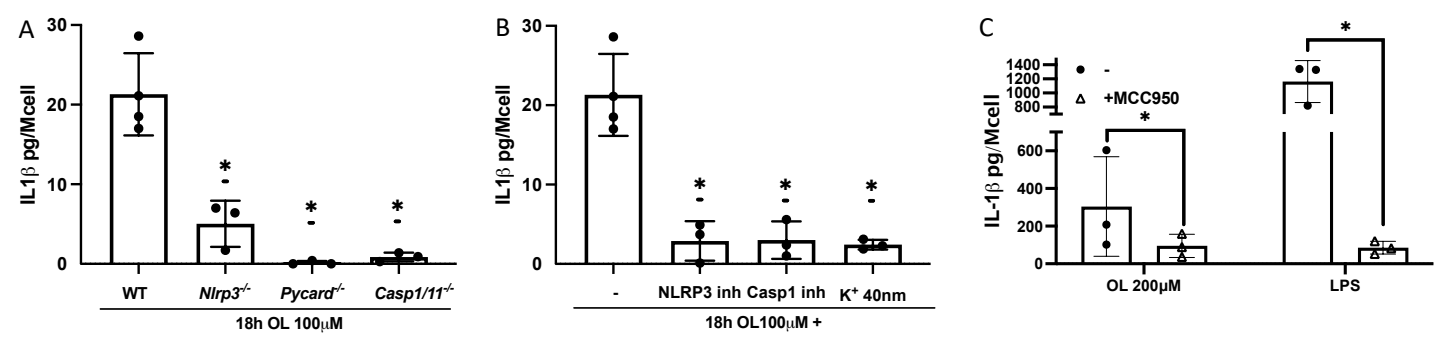

Figure 4: OL activates the NLRP3 inflammasome in murine macrophages and human mononuclear

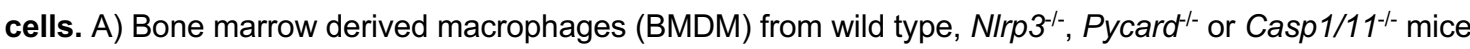
were incubated for $18 \mathrm{~h}$ with OL $(100 \mu \mathrm{M})$ and IL-1 $\beta$ was quantified in cell supernatants by ELISA. B) BMDM from wild type mice were incubated for $18 \mathrm{~h}$ with $\mathrm{OL}(100 \mu \mathrm{M})$ in the absence or in the presence of the NLRP3 inhibitor MCC950 $(10 \mu \mathrm{M})$, the caspase-1 inhibitor VX-765 (10 $\mu \mathrm{M})$ or in a high $\mathrm{K}^{+}$buffer $(40$ $\mathrm{mM} \mathrm{KCl}$ ) to impair $\mathrm{K}^{+}$efflux. IL-1 $\beta$ was quantified in cell supernatants by ELISA. C) Human peripheral blood derived mononuclear cells from healthy donors were incubated for $18 \mathrm{~h}$ without (Ctrl) or with LPS $(100 \mathrm{ng} / \mathrm{mL})$ or OL $(200 \mu \mathrm{M})$ in the absence or in the presence of MCC950 (10 $\mu \mathrm{M})$. IL-1 $\beta$ was quantified in cell supernatants by ELISA. Bars represent the mean of three or more independent experiments represented by dots $(N=3$ to 4$)$. Statistical analysis: Paired $t$-test $(A, B)$ or ratio $t$-test $(C)$, significant difference with respect to the condition with $\mathrm{OL}$ in the absence of inhibitors $(B, C)$ or in wild type BMDM (A) for $p<0.05\left(^{*}\right)$. 


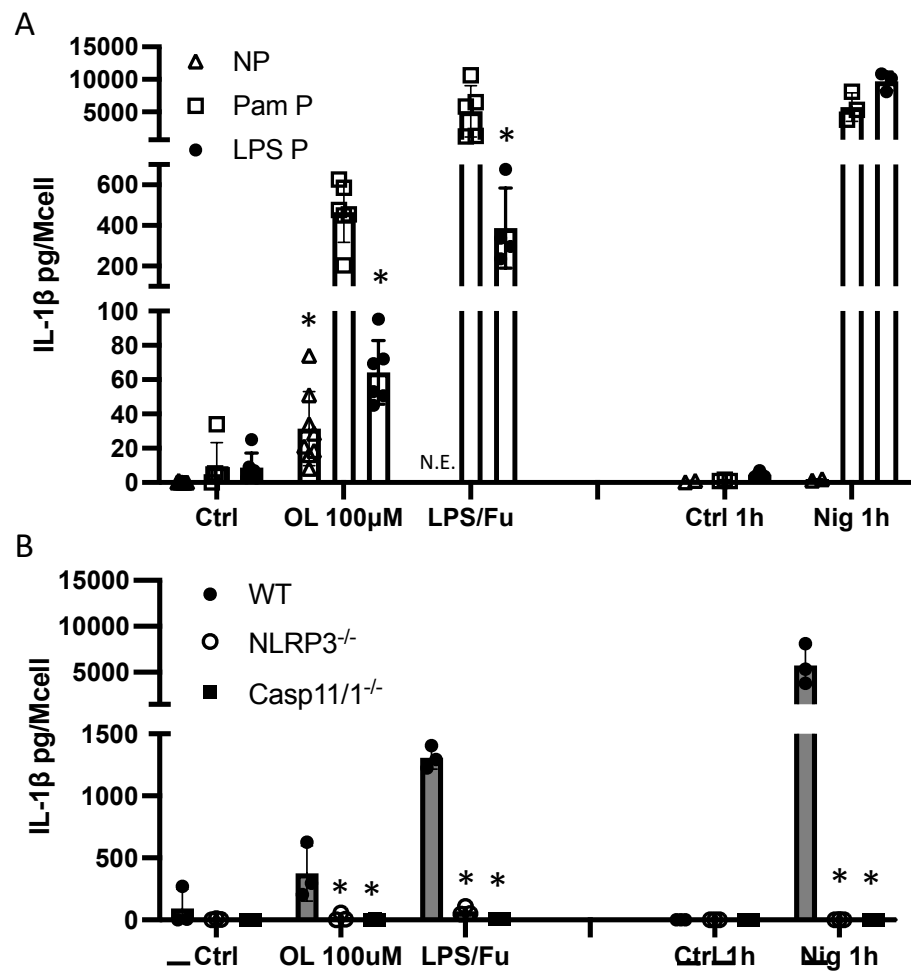

Figure 5: Pam3CSK4 priming enhances both OL- and LPS/Fu-induced but not nigericin-induced IL-

$1 \beta$ secretion. A) Bone marrow derived macrophages (BMDM) from wild type mice were incubated for $4 \mathrm{~h}$ without (NP) or with Pam3CSK4 $(1 \mu \mathrm{g} / \mathrm{mL}$, Pam P) or LPS $(1 \mu \mathrm{g} / \mathrm{mL}$, LPS P), then washed and further incubated for $18 \mathrm{~h}$ without (Ctrl) or with OL $(100 \mu \mathrm{M})$ or LPS/Fu $(1 \mu \mathrm{g} / \mathrm{mL}$ LPS and $0,5 \%$ Fugene), or incubated for $1 \mathrm{~h}$ without (Ctrl) or with nigericin (10 $\mu \mathrm{M}, \mathrm{Nig})$. IL-1 $\beta$ was quantified in cell supernatants by ELISA; N.E.: not evaluated. B) BMDM from wild type, NIrp3 $3^{--}$, or Casp1/11 $1^{-/-}$mice were incubated for $4 \mathrm{~h}$ with Pam3CSK4 $(1 \mu \mathrm{g} / \mathrm{mL})$, then washed and incubated for $18 \mathrm{~h}$ without (Ctrl) or with OL $(100 \mu \mathrm{M})$ or LPS/Fu ( $1 \mu \mathrm{g} / \mathrm{mL}$ LPS and 0,5\% Fugene), or incubated for $1 \mathrm{~h}$ without (Ctrl) or with nigericin (10 $\mu \mathrm{M}, \mathrm{Nig})$. Bars represent the mean of more than three experiments represented as dots $(\mathrm{N}=3$ to 8$)$. Statistical analysis: Paired $t$-test, significant difference with respect to the Pam3CSK4 primed condition in wild-type BMDMs for $p<0.05\left(^{*}\right)$. 


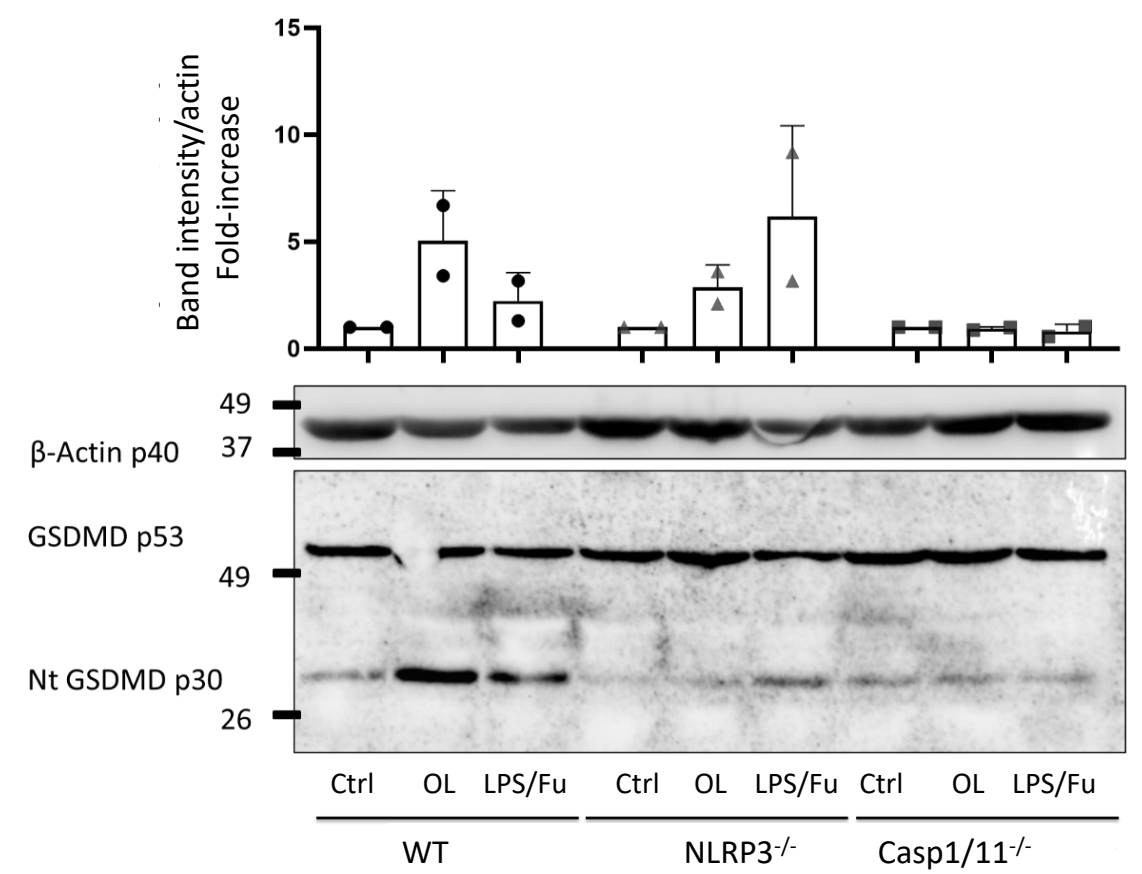

Figure 6: OL induces GSMD cleavage is not dependent on NLRP3. Bone marrow derived

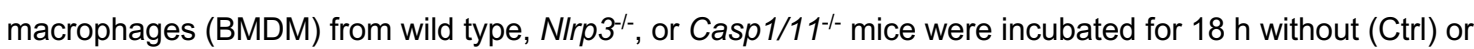
with OL $(100 \mu \mathrm{M})$ or LPS/Fu (1 $\mu \mathrm{g} / \mathrm{mL}$ LPS and 0,5\% Fugene). Cell lysates were immunoblotted for GSDMD or $\beta$-actin (used as charge control). The quantification of cleaved GSDMD normalized to $\beta$-actin represented as fold-increase with respect to the Ctrl is shown on the top. Bars represent the mean of two experiments represented by different symbols. Western blot on the bottom is representative of two independent experiments.
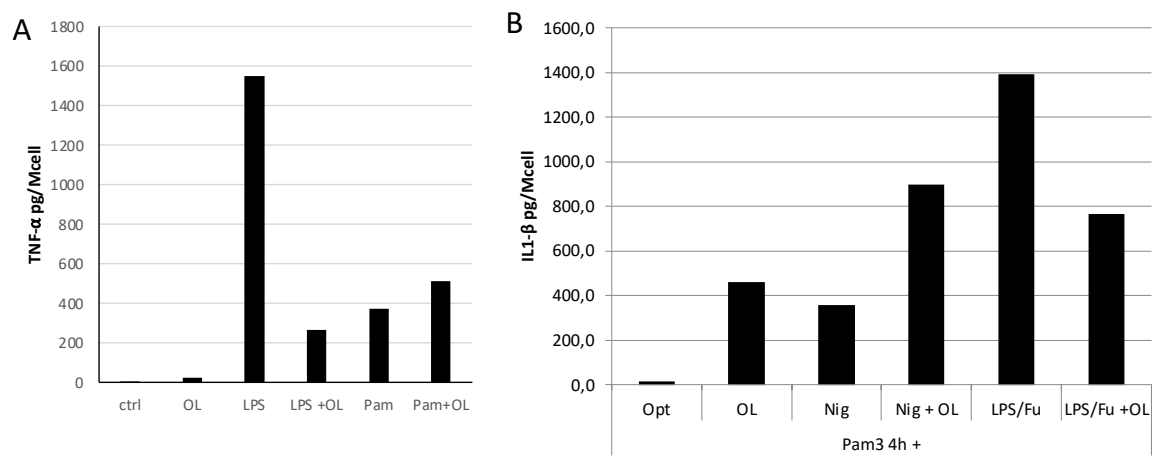

Figure 7: OL inhibits LPS-induced TLR4 and NLRP3 inflammasome activation but not Pam $\mathrm{CSK}_{4}$ induced TLR2 activation and nigericin-induced NLRP3 inflammasome activation. A) Bone marrow derived macrophages (BMDM) from wild type mice were incubated for $4 \mathrm{~h}$ without (Ctrl) or with OL (100 $\mu \mathrm{M}), \mathrm{Pam}_{3} \mathrm{CSK}_{4}(1 \mu \mathrm{g} / \mathrm{mL})$ or LPS $(100 \mathrm{ng} / \mathrm{mL})$ in the absence or in the presence of OL $(100 \mu \mathrm{M},+\mathrm{OL})$. TNF- $\alpha$ was quantified in cell supernatants by ELISA. B) BMDM from wild type mice were incubated for $4 \mathrm{~h}$ with Pam3CSK4 $(1 \mu \mathrm{g} / \mathrm{mL})$, then washed and incubated for $18 \mathrm{~h}$ without (Ctrl) or OL (100 $\mu \mathrm{M})$ or LPS/Fu $(1 \mu \mathrm{g} / \mathrm{mL}$ LPS and 0,5\% Fugene) in the absence or in the presence of OL (100 $\mu \mathrm{M}, \mathrm{LPS} / \mathrm{Fu}+\mathrm{OL})$ or for 18 $\mathrm{h}$ without or with $\mathrm{OL}(100 \mu \mathrm{M})$ and then for $1 \mathrm{~h}$ with nigericin $(10 \mu \mathrm{M}$, Nig and Nig+OL). IL-1 $\beta$ was quantified in cell supernatants by ELISA. Data are representative of one experiment. 